INPLASY

PROTOCOL

To cite: Zeng et al. The effects of Swimming Sports on the prevention and restoration of COVID-19 and its variant strains: A study protocol systematic review. Inplasy protocol 2021120075. doi: 10.37766/inplasy2021.12.0075

Received: 16 December 2021

Published: 16 December 2021

Corresponding author:

Qing Liu

121919626@qq.com

Author Affiliation:

Chengdu Sport University.

Support: 21XJC890001.

Review Stage at time of this submission: Completed but not published.

Conflicts of interest: None declared.

\section{The effects of Swimming Sports on the prevention and restoration of COVID-19 and its variant strains: A study protocol systematic review}

Zeng, J1; Wang, Y2; Yang, Q3; Shang, Y4; Zeng, Z5; Liu, Q6.

Review question / Objective: From previous studies, we found that in addition to basic medical treatment, swimming with a certain amount of load and intensity can promote the ventilator of the human body, thereby playing an auxiliary and preventive role in the treatment of COVID-19 and its variant strains.

Condition being studied: The effects of Swimming Sports on the prevention and restoration of COVID-19 and its variant strains. Two researchers independently screened and extracted the literature, and evaluated the bias risk of the included studies. The methodological quality of the included literature was evaluated by the Cochrane bias risk assessment tool.

INPLASY registration number: This protocol was registered with the International Platform of Registered Systematic Review and Meta-Analysis Protocols (INPLASY) on 16 December 2021 and was last updated on 16 December 2021 (registration number INPLASY2021120075).

\section{INTRODUCTION}

Review question / Objective: From previous studies, we found that in addition to basic medical treatment, swimming with a certain amount of load and intensity can promote the ventilator of the human body, thereby playing an auxiliary and preventive role in the treatment of COVID-19 and its variant strains.

Condition being studied: The effects of Swimming Sports on the prevention and restoration of COVID-19 and its variant strains. Two researchers independently screened and extracted the literature, and 
evaluated the bias risk of the included studies. The methodological quality of the included literature was evaluated by the Cochrane bias risk assessment tool.

\section{METHODS}

Search strategy: COVID-19; Delta; Omicron; Swimming Sport; Systematic reviewChina knowledge net work, Web of science, Google scholar, PubMed database.

\section{Participant or population: Not reported.}

Intervention: The experimental group received medical treatments and Swimming Sports intervention scheme after medical treatments.

Comparator: The control group only received the medical treatments.

Study designs to be included: Randomized controlled trial.

Eligibility criteria: This study searched China knowledge net work, Web of science, Google scholar, PubMed database to search for the relevant research on swimming prevention and treatment for COVID-19, and the deadline for searching was December 2021.

Information sources: China knowledge net work, Web of science, Google scholar, PubMed database.

Main outcome(s): through swimming to improve lung function, so as to achieve the prevention of infection with COVID-19 and the recovery effect after the cure, is conducive to the COVID-19 and its variants by people all over the world.

Quality assessment / Risk of bias analysis: Due to the extremely variant strain of COVID-19, a virus for the whole world, we should pay attention to non English language analysis in order to ensure the accuracy of the conclusion, so that other scholars can further study COVID-19.

Strategy of data synthesis: Heterogeneity analysis test is to test various potential intra group and inter group differences and different qualitative conditions that affect the effect quantity. The usual test method is 12 or q test. In this study, 12 statistic is used for test, and the test formula is: $12=$ $[Q-(k-1) /$ q] $\times 100 \%$ 。When $12<50 \%$, $P \geq 0.1$, it is considered that there is no obvious heterogeneity among various studies. At this time, the fixed effects model is used to calculate and combine the effects; If $\mathrm{I} 2>50 \%, \mathrm{P}<0.1$, it is considered that the heterogeneity is high. At this time, further heterogeneity source analysis will be carried out. Generally, subgroup analysis or sensitivity analysis will be used, and the literature will be eliminated one by one to observe the heterogeneity, and random effects model will be used to merge the effects. However, the Cochrane reviewers' Handbook believes that comparing the differences between subgroups is indeed helpful to understand the regulatory variables, but the essence of subgroup analysis is observational, and it does not strictly control other interfering variables. Therefore, subgroup analysis has certain limitations. When conducting subgroup analysis, the conclusion of subgroup effect should be carefully explained, Finally, $P$ value combined with $95 \% \mathrm{Cl}$ was selected as the effect quantity for statistical analysis. In addition, according to Cohen, 0.2 is a small effect quantity; 0.5 is the medium effect quantity; 0.8 is a large effect.

Subgroup analysis: According to the results of meta analysis and the purpose of this study, subgroup analysis of regulatory variables was carried out to further explore the source of heterogeneity and compare the effect amount between subgroups.

Sensitivity analysis: Sensitivity analysis is used to analyze research quality, intervention method ,publishing type, and so on. If there is large heterogeneity, sensitivity analysis should be carried out. The sensitivity analysis adopts the method of eliminating the literature one by one.

Country(ies) involved: China. 
Keywords: COVID-19; Delta; Omicron; Swimming Sport; Systematic review study protocol systematic review.

Contributions of each author:

Author 1 - Jing Zeng - Conceptualization.

Email: 1208676945@qq.com

Author 2 - Yang Wang.

Email: 416775014@qq.com

Author 3 - Qunru Yang.

Email: 29742999@qq.com

Author 4 - Yao Shang.

Email: 747378705@qq.com

Author 5 - Zhengfang Zeng.

Email: 418061907@qq.com

Author 6 - Qing Liu.

Email: 121919626@qq.com 